\title{
Disruption of Rankl/Rank Signaling Reduces TNF-Induced Joint Inflam- mation In Vivo
}

\author{
Zhenqiang Yao ${ }^{1}$, Ping Li $^{2}$, Qian Zhang ${ }^{1}$, Ruolin Guo ${ }^{1}$, Edward M. Schwarz ${ }^{2}$, Brendan F. Boyce ${ }^{1,2}$ \\ and Lianping Xing*,1,2 \\ ${ }^{1}$ Department of Pathology and Laboratory Medicine, and ${ }^{2}$ Center for Musculoskeletal Research, University of Rochester \\ Medical Center, Rochester, NY 14642, USA
}

\begin{abstract}
TNF is a dominant inflammatory cytokine that is over-produced in the joints of patients with rheumatoid arthritis, as well as animal models of this disease. It promotes bone destruction and inflammation through stimulation of osteoclast formation and activation. While it has been established that the RANKL/RANK system is essential for TNF-induced osteoclast formation and bone erosion in vivo, its role in TNF-induced inflammation remains poorly understood and controversial. Here, we report that genetic and biologic disruption of the RANKL/RANK signaling eliminates knee joint inflammation in TNF-Tg/RANKL ${ }^{-/-}$, TNF-Tg/RANK ${ }^{-/}$and RANK:Fc treated-TNF-Tg mice as determined by functional assessments and histomorphometry. Among several potential mechanisms that could be responsible for this decrease in inflammation, we found that RANKL synergizes with TNF to stimulate TNF production by osteoclast precursors. Thus, blockade of RANKL/RANK signaling may be a viable therapeutic strategy to prevent inflammation and bone loss in diseases where elevated TNF is a key pathogenic factor.
\end{abstract}

\section{INTRODUCTION}

The receptor activator of NF- $\mathrm{\kappa B}$ ligand (RANKL)/ RANK system is essential for osteoclast formation. RANKL was initially characterized as a T-lymphocyte-specific protein [1], and subsequently was detected in bone marrow stromal cells and osteoblasts [2,3]. Later studies demonstrated that RANKL is produced by almost all cell types in the body, including endothelial cells and chondrocytes [4]. Its expression by these cells increases in response to a variety of factors, including cytokines, growth factors and hormones to induce osteoclast formation, activation and survival in normal and disease states [5-7]. In contrast to the broad expression of RANKL, RANK, the receptor for RANKL, is only identified on mature osteoclasts, dendritic cells, and precursor cells of osteoclasts and dendritic cells [4, 8, 9], which are therefore, the major target cells for RANKL. RANK expressing cells appear not to produce RANKL, thus the signal is transduced through a paracrine mechanism.

Despite the essential role of RANKL in the differentiation of osteoclast precursors (OCPs) to mature osteoclasts, little is known as to whether or not it affects other functions of these cells. Recently, several studies have demonstrated that RANKL treatment stimulates OCPs or osteoclasts to produce various cytokines and chemokines, including IL-1, TNF and MCP-1 [10-13], indicating that the RANKL/RANK system is not only critical for osteoclastogenesis to resorb bones, but also important for their activation and effector cell function.

*Address correspondence to this author at the Department of Pathology and Laboratory Medicine, University of Rochester Medical Center, 601 Elmwood Avenue, Box 626, Rochester, New York, 14642, USA; Tel: 585273-4090; Fax: 585-756-4468; E-mail: lianping_xing@urmc.rochester.edu
The importance of this RANKL signal in osteoclasts and OCPs towards their activation and contribution to the pathogenesis of inflammatory arthritis has not been addressed because earlier studies failed to detect effects on inflammation. For example, osteoprotegerin (OPG):Fc treatment prevented bone loss and cartilage destruction, but had no discernible effect on inflammation in adjuvant-induced arthritis in Lewis rats [14]. In serum transfer induced arthritis, $\mathrm{RANKL}^{-/}$mice develop a comparable degree of inflammation to wild type (wt) littermates, but bone erosion was greatly reduced [15]. Thus, the current paradigm is that RANKL-mediated OCP differentiation is critical for bone erosion, but inflammation is either independent of RANKL signaling, or is mediated by several signaling pathways which have overlapping functions in vivo.

During the course of studying the role of the RANKL/ RANK signaling in TNF-mediated osteoclastogenesis in vivo, we generated $\mathrm{TNF}-\mathrm{Tg} / \mathrm{RANK}^{-/}$hybrid mice. To our surprise, these mice not only had no bone erosion [16], but also had no or slight inflammation in their knee joints. To further elucidate the effect of RANKL blockade on TNFinduced arthritis and the mechanisms involved, we generated TNF-Tg/RANKL ${ }^{-/}$mice and found that they also had little to no knee joint inflammation. More importantly, RANK:Fc treatment of TNF-Tg mice also significantly reduced the severity of their knee joint inflammation. In vitro, we found that RANKL and TNF synergistically stimulate TNF production by OCPs, indicating that blockade of the RANKL/ RANK pathway may prevent joint inflammation in TNFinduced arthritis through inhibition of OCP activation.

\section{EXPERIMENTAL PROCEDURES}

\section{Animals}

The 3647 TNF-Tg were originally obtained from Dr. G. Kollias [17]. TNF-Tg/RANK ${ }^{-/}$mice were characterized pre- 
viously $[16,18]$. $\mathrm{RANKL}^{-/-}$mice in a $\mathrm{C} 57 \mathrm{Bl} / 6$ background were provided Dr. S.C. Marks [19] and used to produce the TNF-Tg/RANKL ${ }^{-1-}$ mice through mating. KRN-TCR-Tg mice were obtained from Drs. Mathis and C. Benoist [20]. $\mathrm{K} / \mathrm{BxN}$ mice were generated by breeding KRN-TCR-Tg mice with nonobese diabetic mice (The Jackson Laboratory, Bar Harbor, ME). All animal studies were approved by the Institutional Animal Care and Use Committee of Rochester University.

\section{Arthritis Models in RANKL Blockade Background and Clinical Assessment}

Three models were used. 1) TNF-Tg/RANKL ${ }^{-1-}$ mice were generated by intercrossing TNF-Tg and RANKL ${ }^{+-}$ mice. Clinical evaluation was performed once a week, start- ing at 10 week of age in TNF-Tg $(\mathrm{N}=21)$ and TNF$\mathrm{Tg} / \mathrm{RANKL}^{-1-}(\mathrm{N}=5)$ mice for 8 weeks. Paw swelling and grip strength scores were evaluated as reported previously [21]. The deformation score was used because we observed that the first easily recognized sign in TNF-Tg mice is joint deformation, including toe flexion, contraction and shortening. The severity of joint deformation is defined as 4 scores: 0-no deformation of toes and ankle, 1-mild, 2-moderate, 3severe deformation. 2) Eight-week-old $\mathrm{RANK}^{-1-}$ mice and $\mathrm{RANK}^{+/-}$littermates $(\mathrm{N}=3$ per genotype) were injected with serum obtained from arthritic $\mathrm{K} / \mathrm{BxN}$ mice $(10 \mu \mathrm{l} / \mathrm{gram}$ of body weight, i.p.) on day 0 and day 2 . Paw swelling was observed once a day for 3 weeks. 3) Ten-week-old TNF-Tg mice ( $\mathrm{N}=8$ per group) were given RANK:Fc $(10 \mathrm{mg} / \mathrm{kg}$, i.p.) or vehicle three time a week for 8 weeks. Leg bones were
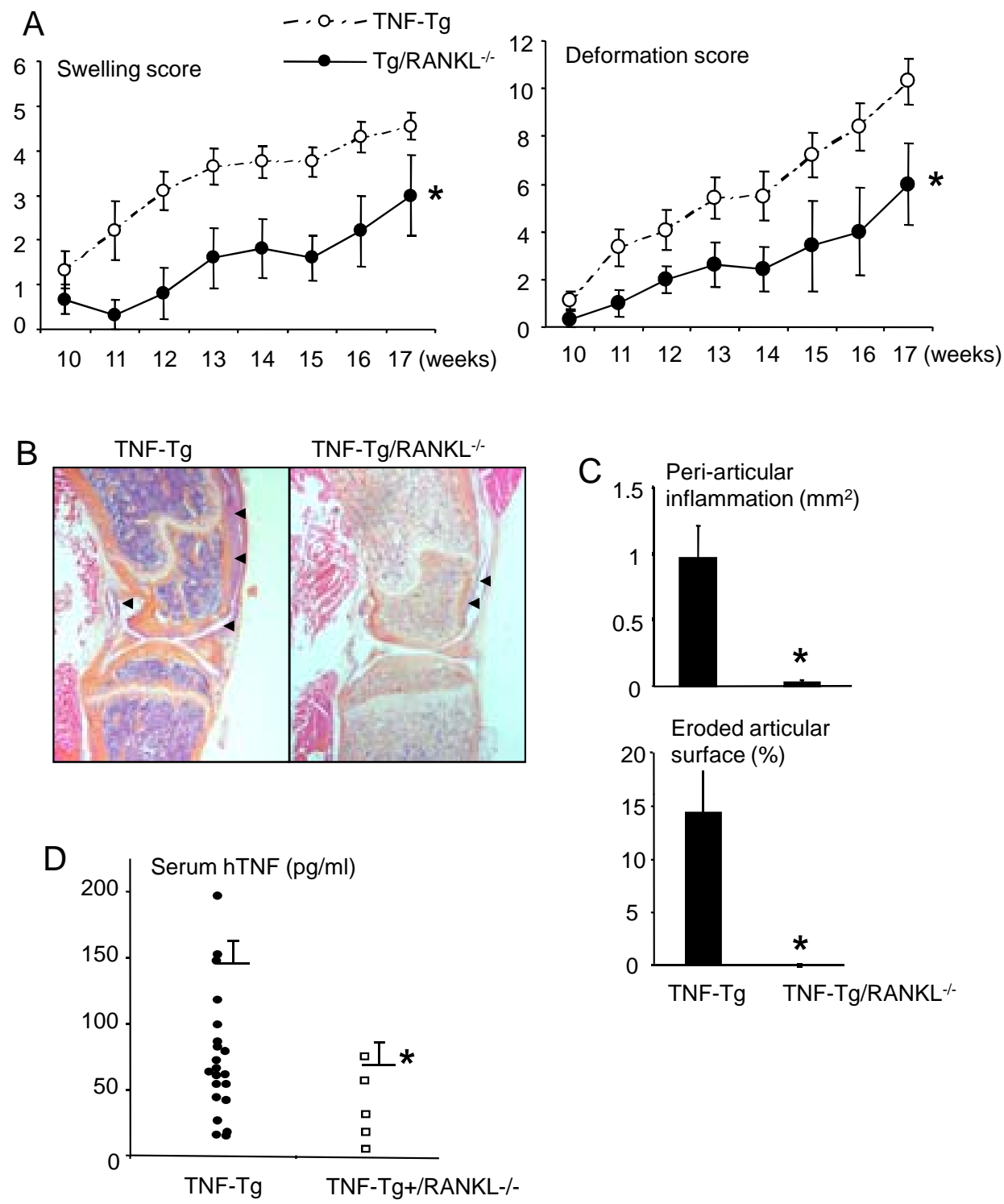

Fig. (1). Significantly reduced synovial inflammation in joints of TNF-Tg/RANKL ${ }^{-/-}$hybrid mice. (A) The severity of arthritis in TNF$\mathrm{Tg}$ and TNF-Tg/RANKL ${ }^{-1-}$ hybrid mice was evaluated by swelling and joint deformation. Animals were examined once a week from 10week-old until 17-week-old. (B) Representatives of H\&E-stained knee joint sections from a TNF-Tg and a TNF-Tg/RANKL ${ }^{-/-} \mathrm{mouse}(2 \mathrm{X}$ magnification). The synovial tissue is indicated by black arrow heads. (C) Histomorphometric analyses of knee joint sections were performed using Osteometric software. Values are presented as the mean plus SEM of 5 TNF-Tg/RANKL ${ }^{-/}$mice and 21 TNF-Tg mice from the same litters. (D) Serum human TNF transgene concentration in TNF-Tg and TNF-Tg/RANKL ${ }^{-/}$mice was measured by ELISA. Dot plot shows the value of individual mouse. ${ }^{*} p<0.05$ vs TNF-Tg mice. 
harvested for histology and blood was used for measurement of hTNF levels by the end of experiments.

\section{Histology}

Paraffin sections were stained with H\&E or for TRAP activity. Histomorphometric analysis were performed using an OsteoMeasure program (Osteometrics, Atlanta, GA) [18]. Peri-articular inflammation $\left(\mathrm{mm}^{2}\right)$ and eroded articular surface $(\%)$ were measured around knee joints and the number of osteoclast per millimeter bone surface was countered in the proximal tibiae region.

\section{Osteoclast Precursor Cultures and Quantitative Real-} Time RT-PCR

Splenocytes from WT and $\mathrm{RANK}^{-/-}$mice were cultured with M-CSF for 3 days to generate OCPs as described previously [22]. Cells were treated with various amounts of RANKL, TNF or in combination; and the murine $T N F$ and $R A N K L$ mRNA expression levels were determined by real time RT-PCR analysis as described previously [22].

\section{Statistical Analysis}

Data are shown as mean \pm SEM. Group mean values were compared by the Mann-Whitney $U$-test for nonNormal distributed data and ANOVA flowed by student $t$ test for normal distributed data.

\section{RESULTS AND DISCUSSION}

\section{RANKL Blockade Prevents Joint Inflammation of TNF- Tg Mice}

To explore the possibility that RANKL/RANK signaling is involved in arthritis, we compared joint phenotypes of TNF-Tg, RANKL ${ }^{-/-}$, TNF-Tg/RANKL ${ }^{-/-}$and wt mice. The gross appearance, movement, and strength of TNF$\mathrm{Tg} / \mathrm{RANKL}^{-/-}$mice were similar to that of $\mathrm{RANKL}^{-/-}$mice, but markedly improved compared to TNF-Tg mice (Fig. 1A). As expected, all TNF-Tg mice (21/21) generated from the same mating developed joint inflammation that was readily apparent from histology (Fig. 1B). Also as predicted, all TNF-Tg/RANKL ${ }^{-/}$mice (5/5) develop severe osteopetrosis due to a lack of TRAP $^{+}$osteoclast formation (Fig. 1B). However, 4 of 5 TNF-Tg/RANKL ${ }^{-/-}$mice had no knee joint inflammation and their joint structures were the same as $\mathrm{RANKL}^{-/-}$mice by histology (Fig. 1C). One TNF-Tg/ RANKL $^{-/-}$mouse had a slightly thinker synovium (Fig. 1B). The serum hTNF levels in TNF-Tg/RANKL ${ }^{-/}$mice were significantly lower than those of TNF-Tg mice (Fig. 1D). Murine TNF levels from blood of these mice were undetectable using a commercial available TNF ELISA kit [18].

To confirm the role of RANKL/RANK signaling in TNFinduced inflammation in an independent genetic model and
A
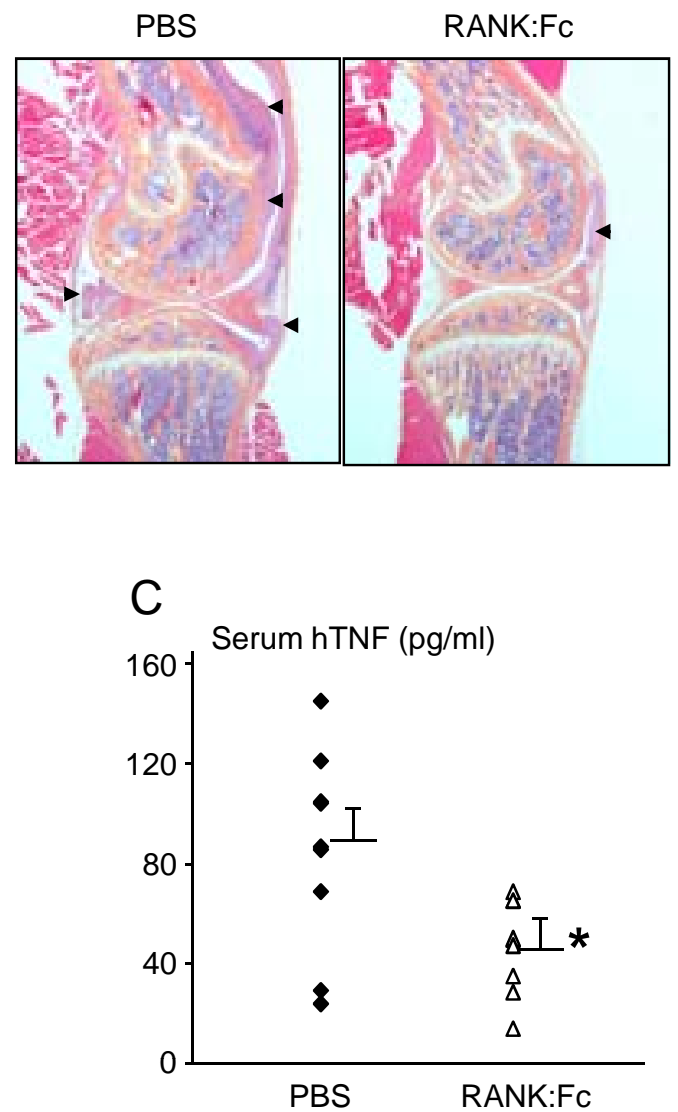

B Peri-articular

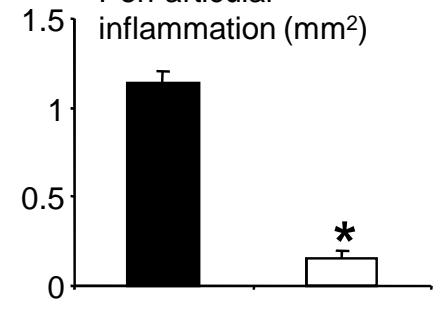

Eroded articular

30 surface (\%)

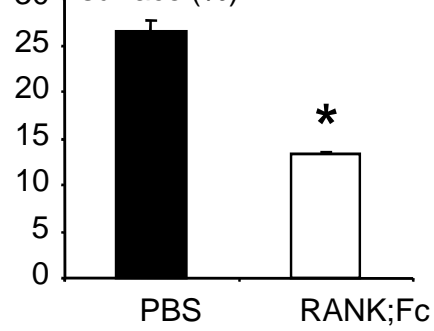

Fig. (2). RANK:Fc-treated TNF-Tg mice have reduced synovial inflammation in joints. Ten-week-old TNF-Tg were treated with RANK:Fc or PBS for 2 months. (A) Representatives of H\&E-stained sections from a PBS-treated TNF-Tg and a RANK:Fc-treated TNF-Tg mouse (2X magnification). The area of synovial tissue was indicated by black arrow heads. (B) Histomorphometric analyses of knee joint sections and (C) serum human TNF transgene concentration were measured as described in Fig. (1). Values are presented as the mean plus SEM of 8 mice per group. ${ }^{*} p<0.05$ vs PBS-treated TNF-Tg mice. 
in non-osteopetrotic mice, we analyzed bone sections from 4-month-old TNF-Tg/RANK ${ }^{-/}$mice $(\mathrm{N}=3)$ as well as from RANK:Fc treated TNF-Tg mice $(\mathrm{N}=8)$, which received RANK:Fc for 8 weeks from 10-week-old when the arthritis like symptoms just begin. Similar to TNF-Tg/RANKL ${ }^{-/}$ mice, TNF-Tg/RANK ${ }^{-/}$mice had neither joint inflammation nor bone erosion. RANK:Fc treatment significantly decreased the inflammatory area in the knee joints of all 8 TNF-Tg mice, compared to vehicle-treated TNF-Tg mice. Similar to TNF-Tg/RANKL ${ }^{-/-}$mice, RANK-Fc treated mice had decreased serum hTNF levels than PBS-treated mice (Fig. 2). Together, our data definitively show that genetic disruption of RANKL signaling from birth abolishes, while inhibition of RANKL at the onset of inflammatory arthritis significantly diminishes TNF-induced joint inflammation.

Since our findings differ from previous publications in which the blockade of RANKL signaling pathway by
OPG:Fc or the induction of arthritis by serum transfer in RANKL $^{-/-}$mice does not prevent joint inflammation $[14,15$, 23], we used another model to test if RANKL blockade only benefits TNF-induced arthritis. In these experiments we induced arthritis by injecting arthritic serum from $\mathrm{K} / \mathrm{BxN}$ mice into $\mathrm{RANK}^{-/-}$or $\mathrm{RANK}^{+/-}$mice, and measured paw thickness daily starting one day after the last serum injection. As reported in $\mathrm{RANKL}^{-/-}$mice using the same model [15], both $\mathrm{RANK}^{-/-}$and $\mathrm{RANK}^{+/-}$mice developed a similar degree of joint redness and swelling (data not shown). Histomorphometric analysis of the ankle joints of these mice confirmed the similar severity of inflammation (mean +/- SEM of the percentage of inflammation area in knee joints: $5.6+/-$ 1.16 in $\mathrm{RANK}^{+/-}$mice vs $7.7+/-0.34$ in $\mathrm{RANK}^{-/-}$mice, $\mathrm{N}=3$ mice per genotype, $p>0.05)$. Thus, the RANKL/RANK dependent effects on inflammation appear to be selective for TNF-induced arthritis.
A

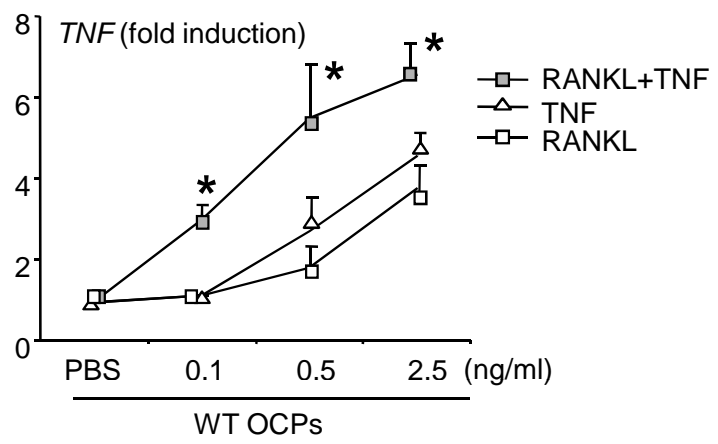

B

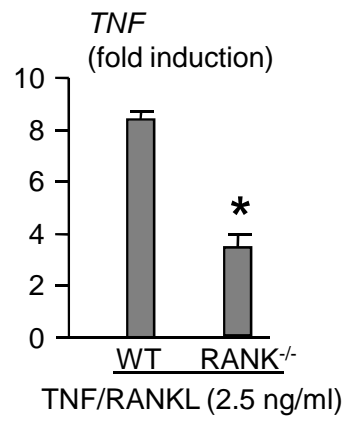

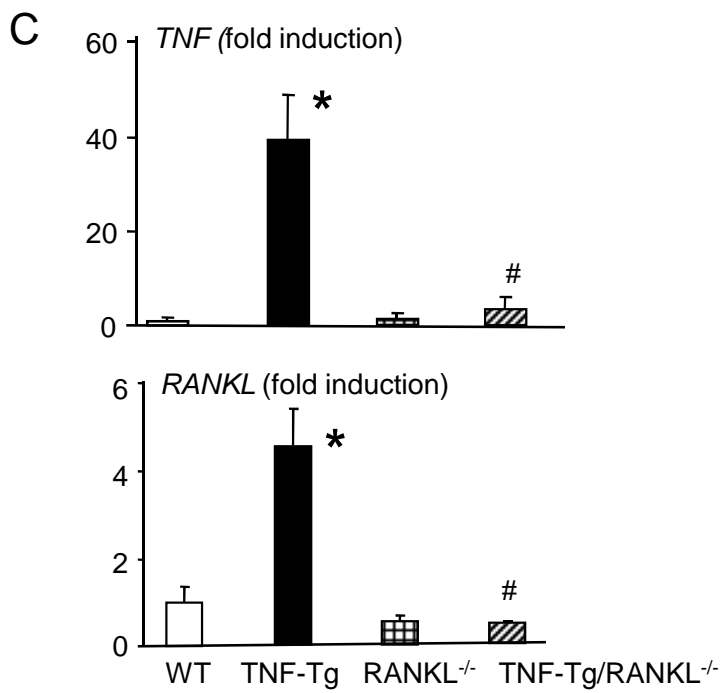

Fig. (3). RANKL and TNF synergistically stimulate $T N F$ mRNA expression by osteoclast precursor cells and decreased $R A N K L$ and TNF levels in joints of TNF-Tg/RANKL ${ }^{-/}$mice. (A) Spleen cells from WT mice were cultured with M-CSF (10 ng/ml) for 3 days to generate osteoclast precursors and then were treated with various amount of TNF, RANKL or in combination for 1 hour. (B) Spleen cells from WT and RANK ${ }^{-1-}$ mice were cultured with M-CSF and treated with TNF+RANKL $(2.5 \mathrm{ng} / \mathrm{ml})$ for 1 hour. Murine TNF mRNA expression was examined by real time RT-PCR. Values are presented as the mean plus SEM of 3 independent experiments done in triplicate, where the PBS control is arbitrarily set at 1 . Similar results were obtained from an additional two pairs of WT and RANK ${ }^{-/-}$mice. $($C) Four-month-old WT, TNF-Tg, RANKL ${ }^{-/-}$, and TNF-Tg/RANKL ${ }^{-/}$mice from the same litter were sacrificed and total RNA was extracted from wrist joints. Expression levels of murine $T N F$ and $R A N K L$ mRNA were measured by real-time RT-PCR. * $p<0.05$ vs WT cells or mice and \#p<0.05 vs TNF-Tg mice. 


\section{RANKL Synergistically with TNF to Stimulate TNF Production by OCPs}

To elucidate the mechanisms by which RANKL blockade inhibits inflammation specifically in TNF-induced arthritis, we reasoned that RANKL must affect a factor that is essential for the initiation of joint inflammation in TNF-induced, but not for the serum-induced arthritis, and that this factor must be produced by RANK expressing cells. We focused on TNF because it is the driving force for the onset of inflammatory arthritis in TNF-Tg mice [17], but not for mice receiving arthritic serum [24].

TNF is produced by many cell types at inflammatory foci, including macrophages, synoviocytes, $\mathrm{T}$ cells, dendritic cells, osteoclasts and OCPs [25]. Among these, only dendritic cells, osteoclasts and OCPs are RANK expressing cells. Since TNF-Tg/c-Fos ${ }^{-1-}$ mice have no osteoclasts, but have elevated serum TNF levels and joint inflammation [21], it is likely that the absence of inflammation in TNF$\mathrm{Tg} / \mathrm{RANK}^{-/-}$or TNF-Tg/RANKL ${ }^{-/-}$mice is not due to a lack of mature osteoclast-produced TNF. To examine if RANKL promotes TNF production by OCPs that also give rise of dendritic cells, we generated OCPs from splenocytes of wt and $\mathrm{RANK}^{-1-}$ mice and treated them with RANKL, TNF or in combination. RANKL synergized with TNF to stimulate TNF production. Importantly, RANKL stimulation at doses 100-fold lower than that required for osteoclast formation, potenicated TNF-induced TNF production (Fig. 3A). This synergistic effect of RANKL and TNF was abolished in RANK $^{-1-}$ cells (Fig. 3B). Thus, a permissive level of RANKL in inflamed joints may be sufficient to stimulate TNFinduced TNF production by OCPs to set-up a positive autocrine loop for inflammation. To test this hypothesis, we examined $R A N K L$ and $T N F$ expression levels in the joints of TNF-Tg/RANKL ${ }^{-1-}$ and TNF-Tg mice. RANKL expression was increased in TNF-Tg, but not in TNF-Tg/RANKL ${ }^{-1}$ mice compared to wt littermates (Fig. 3C). Consistent with a decrease in $R A N K L$ mRNA expression, $T N F$ mRNA expression in TNF-Tg/RANKL ${ }^{-1-}$ mice was remarkably reduced (Fig. 3). Interestingly, serum human TNF transgene concentration was decreased in TNF-Tg/RANKL ${ }^{-1-}$ and RANK:Fctreated TNF-Tg mice compared to control TNF-Tg mice (Figs. 1D and 2C). Currently, the mechanisms by which regulate human TNF transgene expression in TNF-Tg mice are not very clear [17]. Our data suggest that its expression is likely associated to the degree of inflammation.

Based on our in vivo and in vitro findings we propose a model to explain the role of the RANKL/RANK system in TNF-induced arthritis (Fig. 4). In an arthritic joint, overexpressed TNF stimulates accessory cells, including synoviocytes, macrophages and other immune cells, to produce RANKL and TNF. This RANKL and TNF synergistically stimulate $\mathrm{RANK}^{+}$OCPs to produce more TNF, forming a positive feedback loop, which exacerbates the inflammatory process. In this model, RANKL-mediated TNF autoregulation plays a critical role in both the initiation and progression of joint disease because if we block the RANKL/RANK pathway before the hTNF transgene level becomes elevated, such as in TNF-Tg/RANKL ${ }^{--}$or TNF$\mathrm{Tg} / \mathrm{RANK}^{-/-}$mice, joint inflammation will not be initiated.

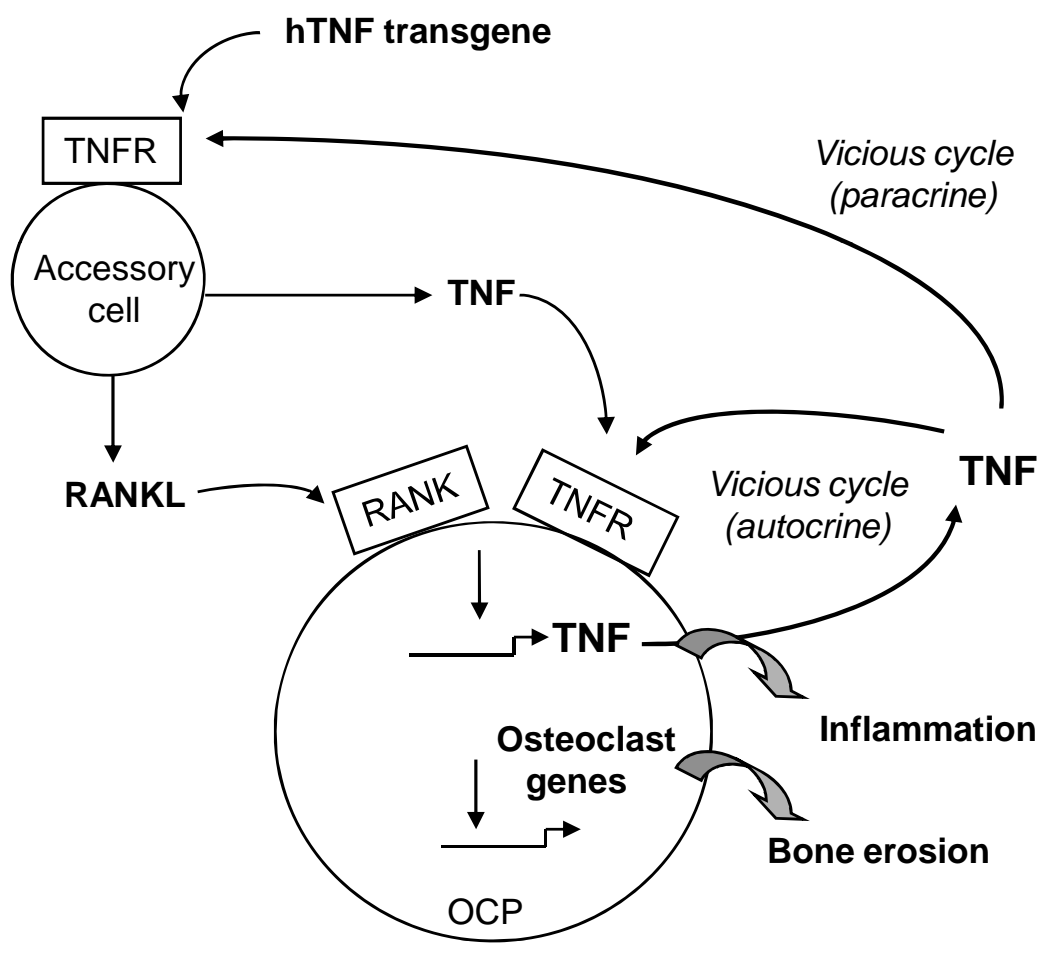

Fig. (4). A model describing the mechanisms by which RANKL blockade inhibits joint inflammation and bone erosion in TNFinduced arthritis. In the inflamed joints, the human TNF transgene stimulates RANKL and TNF production by TNF receptor expressing accessory cells. RANKL then synergizes with exogenous TNF to boost TNF production in RANK and TNF receptor double positive OCPs. This leads to the formation of two vicious cycles: autocrine-between OCPs; and paracrine-between OCPs and accessory cells, which stimulate more TNF and RANKL production. TNF and RANKL trigger OCP activation and differentiation. Thus, OCPs are important effector cells to maintain the inflammation and bone erosion in TNF-induced arthritis. 
However, if RANKL blockade happens after the onset of joint disease at the time when the hTNF transgene levels have been already elevated, such as in RANK:Fc treated 2month-old TNF-Tg mice, the inflammation still exists but to a much lesser degree. This indicates that RANKL-mediated TNF-auto-regulation also plays a considerable role in the effector phase of the disease.

Our findings raise 2 important issues. One is that RANKL blockade may specifically benefit TNF-induced arthritis or certain forms of inflammatory arthritis where $\mathrm{RANK}^{+}$cell-produced cytokines plays a critical role. In support of this, a recent study indicates that RANK:Fc treatment significantly reduces the severity of joint inflammation in LPS-induced arthritis [11]. In this report, the authors demonstrate that RANKL stimulated peripheral blood monocytes (PBMC) to produce cytokines and chemokines, although the dose of RANKL used was very high $(5 \mu \mathrm{g} / \mathrm{ml})$, which is probably due to a low percentage of $\mathrm{RANK}^{+}$cells in PBMC. Whether or not TNF is the initiation factor in this model is not known, but that LPS stimulates TNF production by myeloid cells has been well established [26].

Another issue is how to evaluate results obtained from different animal models of arthritis, because our results strongly argue that using different animal models could result in completely different outcomes in terms of RANKL blockade. This phenomenon is not totally surprising given the variety of mechanisms of actions to induce arthritis in small animals [27]. For instance, $\mathrm{K} / \mathrm{BxN}$ and adjuvantinduced arthritis is working through auto-antibodies, while arthritis in TNF-Tg or IL-1-Tg mice is through cytokines despite all the models led to joint diseases in the later phase [27]. RANKL blockade is effective in TNF- (our study) or LPS-induced [11], but not in $\mathrm{K} / \mathrm{BxN}$ serum induced- or adjuvant-induced arthritis $[14,15]$. This strongly suggests that the specificity of the initiation factor is critical.

In summary, we have demonstrated a novel role for RANKL/RANK system in mediating cytokine-induced inflammatory arthritis. Since RA pathogenesis is a very complicated process involving autoimmune reactions, cytokine production, and genetic modifications. Anti-RANKL therapy may be a viable therapeutic strategy to prevent inflammation and bone loss in diseases where elevated TNF or other cytokines are the key pathogenic factors.

\section{FOOTNOTES}

This work was supported by research grants from the National Institutes of Health (PHS AR 48697 to LX and AR43510 to BFB).

\section{ACKNOWLEDGMENTS}

The RANK:Fc used in this study were provided by Amgen Inc. The authors would like to thank Dr. Xiaoyun Zhang for technical assistance with the histology.

\section{REFERENCES}

[1] Wong BR, Rho J, Arron J, et al. TRANCE is a novel ligand of the tumor necrosis factor receptor family that activates c-Jun $\mathrm{N}$ terminal kinase in T cells. J Biol Chem 1997; 272: 25190-4.

[2] Lacey DL, Timms E, Tan HL, et al. Osteoprotegerin ligand is a cytokine that regulates osteoclast differentiation and activation. Cell 1998; 93: 165-76.
Yasuda H, Shima N, Nakagawa N, et al. Osteoclast differentiation factor is a ligand for osteoprotegerin/osteoclastogenesis-inhibitory factor and is identical to TRANCE/RANKL. Proc Natl Acad Sci USA 1998; 95: 3597-602.

[4] Boyle WJ, Simonet WS, Lacey DL. Osteoclast differentiation and activation. Nature 2003; 423: 337-42.

[5] Goldring SR. Inflammatory mediators as essential elements in bone remodeling. Calcif Tissue Int 2003; 73: 97-100.

[6] Teitelbaum SL. Osteoclasts, integrins, and osteoporosis. J Bone Miner Metab 2000; 18: 344-9.

[7] Udagawa N, Kotake S, Kamatani N, Takahashi N, Suda T. The molecular mechanism of osteoclastogenesis in rheumatoid arthritis. Arthritis Res 2002; 4: 281-9.

[8] Teitelbaum SL, Ross FP. Genetic regulation of osteoclast development and function. Nat Rev Genet 2003; 4: 638-49.

[9] Dougall WC, Glaccum M, Charrier K, et al. RANK is essential for osteoclast and lymph node development. Genes Dev 1999; 13: 2412-24.

[10] Cappellen D, Luong-Nguyen NH, Bongiovanni S, Grenet O, Wanke C, Susa M. Transcriptional program of mouse osteoclast differentiation governed by the macrophage colony-stimulating factor and the ligand for the receptor activator of NFkappa B. J Biol Chem 2002; 277: 21971-82.

[11] Seshasayee D, Wang H, Lee WP, et al. A novel in vivo role for osteoprotegerin ligand in activation of monocyte effector function and inflammatory response. J Biol Chem 2004; 279: 30202-9.

[12] Granfar RM, Day CJ, Kim MS, Morrison NA. Optimised real-time quantitative PCR assays for RANKL regulated genes. Mol Cell Probes 2005; 19: 119-26.

[13] Lee SK, Gardner AE, Kalinowski JF, Jastrzebski SL, Lorenzo JA. RANKL-stimulated osteoclast-like cell formation in vitro is partially dependent on endogenous interleukin-1 production. Bone 2006; 38: 678-85.

[14] Kong YY, Feige U, Sarosi I, et al. Activated T cells regulate bone loss and joint destruction in adjuvant arthritis through osteoprotegerin ligand. Nature 1999; 402: 304-9.

[15] Pettit AR, Ji H, von Stechow D, et al. TRANCE/RANKL knockout mice are protected from bone erosion in a serum transfer model of arthritis. Am J Pathol 2001; 159: 1689-99.

[16] Li P, Schwarz EM, O'Keefe RJ, Ma L, Boyce BF, Xing L. RANK signaling is not required for TNFalpha-mediated increase in CD11(hi) osteoclast precursors but is essential for mature osteoclast formation in TNFalpha-mediated inflammatory arthritis. J Bone Miner Res 2004; 19: 207-13.

[17] Keffer J, Probert L, Cazlaris H, et al. Transgenic mice expressing human tumour necrosis factor: a predictive genetic model of arthritis. EMBO J 1991; 10: 4025-31.

[18] Li P, Schwarz EM, O'Keefe RJ, et al. Systemic tumor necrosis factor alpha mediates an increase in peripheral CD11bhigh osteoclast precursors in tumor necrosis factor alpha-transgenic mice. Arthritis Rheum 2004; 50: 265-76.

[19] Odgren PR, Kim N, MacKay CA, Mason-Savas A, Choi Y, Marks $\mathrm{SC}$, Jr. The role of RANKL (TRANCE/TNFSF11), a tumor necrosis factor family member, in skeletal development: effects of gene knockout and transgenic rescue. Connect Tissue Res 2003; 44 (Suppl 1): 264-71.

[20] Kouskoff V, Korganow AS, Duchatelle V, Degott C, Benoist C, Mathis D. Organ-specific disease provoked by systemic autoimmunity. Cell 1996; 87: 811-22.

[21] Redlich K, Hayer S, Ricci R, et al. Osteoclasts are essential for TNF-alpha-mediated joint destruction. J Clin Invest 2002; 110: 1419-27.

[22] Zhang Q, Badell IR, Schwarz EM, et al. Tumor necrosis factor prevents alendronate-induced osteoclast apoptosis in vivo by stimulating Bcl-xL expression through Ets-2. Arthritis Rheum 2005; 52: 2708-18.

[23] Redlich K, Hayer S, Maier A, et al. Tumor necrosis factor alphamediated joint destruction is inhibited by targeting osteoclasts with osteoprotegerin. Arthritis Rheum 2002; 46: 785-92.

[24] Ji H, Pettit A, Ohmura K, et al. Critical roles for interleukin 1 and tumor necrosis factor alpha in antibody-induced arthritis. J Exp Med 2002; 196: 77-85.

[25] Goldring SR. Pathogenesis of bone and cartilage destruction in rheumatoid arthritis. Rheumatology (Oxford) 2003; 42 (Suppl 2): ii11-6. 
[26] Lin WJ, Yeh WC. Implication of Toll-like receptor and tumor necrosis factor alpha signaling in septic shock. Shock 2005; 24: 206-9.
[27] Sakaguchi S, Sakaguchi N. Animal models of arthritis caused by systemic alteration of the immune system. Curr Opin Immunol 2005; 17: 589-94.

Received: December 31, 2008

(C) Yao et al.; Licensee Bentham Open.

This is an open access article licensed under the terms of the Creative Commons Attribution Non-Commercial License (http://creativecommons.org/licenses/by-nc/3.0/) which permits unrestricted, non-commercial use, distribution and reproduction in any medium, provided the work is properly cited. 